\title{
Tailoring health services to the needs of individual communities
}

\author{
JOHN WOMERSLEY AND DOUGLAS McCAULEY \\ From the Greater Glasgow Health Board, 225 Bath Street, Glasgow G2 4JT
}

SUMMARY In order to assess the need for community health services in different neighbourhoods within Greater Glasgow, it was decided to present a wide variety of health information for each community as a set of summary profiles. These profiles clearly demonstrate that the same areas have the highest standardised mortality ratios, the least favourable socioeconomic circumstances, the highest hospital admission rates, and the poorest child health characteristics. The greatest benefit in overall health would be achieved by targeting community resources on these disadvantaged communities. Adoption of this policy should reduce existing inequalities in health, and we argue that such 'positive discrimination' is implied in the formulae used in Great Britain for allocation of revenue expenditure for community services. The health profiles that we describe provide the baseline information necessary to target community services to particular communities according to objective measures, and to evaluate the effectiveness of new and existing methods of health promotion.

For almost 150 years it has been demonstrated that there is marked social class and geographic variation in health and in the provision of health services. ${ }^{1-8}$ The demonstration of social class differences in health is important as a means of monitoring inequality, but data on service utilisation are not usually available on a social class basis, and it is difficult to target health resources to a particular social class. Geographical differences in health, however, are of similar magnitude to social class differences, ${ }^{9}$ and the identification of areas of poor health indicates clearly where resources should be targeted.

Cluster analysis has been used to identify areas of socioeconomic deprivation from census variables. ${ }^{10}$ ACORN (A Classification of Residential Neighbourhoods) is based on this technique and has been used to classify all addresses in the United Kingdom into which one of 30 types of enumeration district they fall. ${ }^{11}$ These methods, however, are not based on any measure of health. One possible way of identifying areas that are likely to require relatively more health services has been described by Jarman; ${ }^{12}$ this involved computing a score based on a set of ten socioeconomic variables derived from what a sample of general practitioners considered (subjectively) to influence their workload most. Scott Samuel later described a score based on the prevalence of permanent sickness in the employed population as recorded in the national census and on ten socioeconomic census variables that were related to this; ${ }^{13}$ this method should provide a more objective indicator measure of health status for communities, and the scores often differed considerably fromo Jarman's in small areas such as wards.

These latter two methods derive composite scores analogous to the identification of priority areas for housing, education, and other action; they are, therefore, limited in that they provide no specific indication of what type of action is required in terms of health. Carstairs has shown that mortality and various measures of morbidity (but not perinatal or infant deaths) are highly correlated with deprivation, but again this does not permit the specific health problems of particular areas to be uniquely identified. ${ }^{14}$ Other workers have selected a specific health variable such as infant hospital admissions for study on a geographical basis within a health authority; ${ }^{15}$ but there would be an advantage in tackling the various health problems in particular small areas collectively rather than individuallly. We seek to demonstrate the use of a wide variety of routinely collected health indicators to present health information for the various communities within the area served by a large health authority in a compact and visually compelling form. In this way it is hoped to persuade managers to redistribute community resources to resolve specific problems in particular communities in order to reduce the obvious inequalities in health. 


\section{Method}

A wide variety of health and health-related information is available by postcode. It was decided to use the postcode sector (eg, GL12.9 LA23.3) as the base for analysis, there being about 130 postcode sectors (average population about 7500 ) within the area served by the Greater Glasgow Health Board. Postcode sectors are not an ideal base for the definition of communities because on occasions they include very heterogeneous populations; however, they are adequate for most purposes.

The following information sources were used:

(1) demographic information, census 1981: This was used to provide a description of the population resident in each postcode sector and denominator data for the calculation of standardised death and hospital discharge rates.

(2) deaths, 1980-2: These are required for the calculation of standardised death rates and were obtained from computer tapes provided by the Registrar General (Scotland).

(3) hospital discharges, 1980-82: These are required for the calculation of standardised hospital discharge rates and were obtained from hospital discharge records on computer tape.

(4) births, 1983: The number of births to residents of each postcode sector is available each year from both the health authority and the Registrar General (Scotland).

(5) child health numerator data, 1983: The child register and immunisation modules of the national child health record system, ${ }^{16}$ together with an associated health visitor module which has been developed in Glasgow, ${ }^{17}$ provide a considerable amount of numerator information which can be related each year to the number of births in each postcode sector.

These data are collated and displayed using a BBC (B) microcomputer or the Apricot range of microcomputers in a way similar to that used for the 'performance indicators' 18 produced by the Department of Health and Social Security. Centile bars are used for the comparison of a variety of variables for any one postcode sector, and a histogram is available to show the distribution of any chosen variable among the different postcode sectors. For the histogram there is also an interrogation facility, so that postcode sectors with particular values can be immediately identified.

\section{Results}

Figures 1 to 5 give the health profile for postcode sector G40.3, which comprises most of Bridgeton, one of the oldest parts of the city of Glasgow. For each variable analysed there is a longitudinal rectangular box with an indicator which shows, on a centile scale, the rating for Bridgeton in relation to all the other postcode sectors within Greater Glasgow. Also given is the range of values for all the postcode sectors and, in parentheses, the actual value for G40.3 itself. The boxes are arranged so that, in general, indicators at or towards the right hand side indicate that the postcode sector has a population with relatively poor health characteristics or socioeconomic circumstances. The figures show that for Bridgeton most of the indicators are well to the right side, indicating that in health as well as in socioeconomic terms this postcode sector is one of the most disadvantaged in the Greater Glasgow area.

Figure 1 shows that Bridgeton is in the highest decile of all postcode sectors for the proportion of the population that is permanently sick $(5 \%)$, in social classes IV and V (28\%), unemployed (35\% for men), and without a car (88\%). The area also has above the Greater Glasgow average for the proportion of elderly in the population (5\% aged 75 years and over), persons born outwith the UK (although only $1 \%$ ), and local authority housing $(77 \%)$. It is well below average for the proportion of preschool children in the

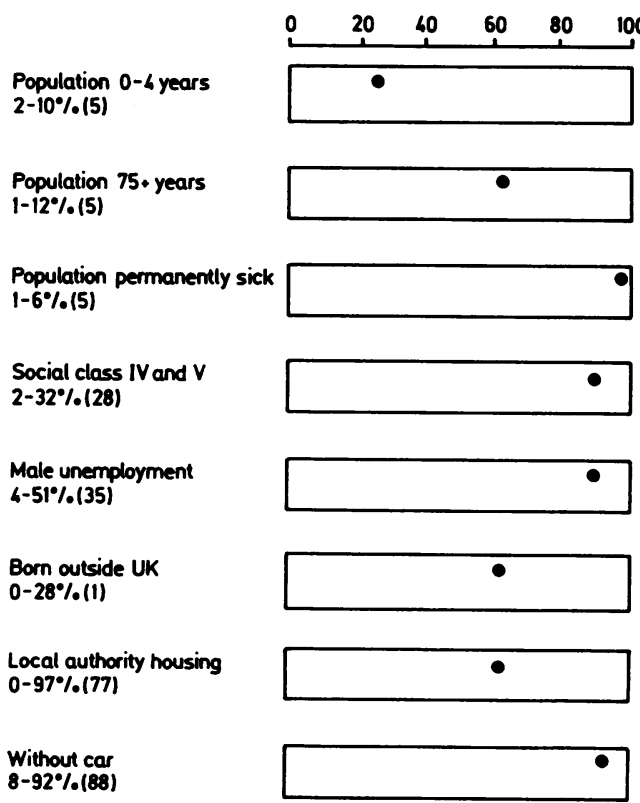

Fig 1 Analysis of eight census variables for Bridgeton. The percentage values give the range for all postcode sectors in Greater Glasgow, the value for Bridgeton being given in parentheses. The indicator in the rectangle gives, on a centile scale, the ranking for Bridgeton in relation to all the postcode sectors. 
192

population $(5 \%)$. The range of values is also of interest: male unemployment in the various postcode sectors within the GGHB area, for example, varied (in 1981 ) from $4 \%$ to $51 \%$, and the proportion of persons in social classes IV and V from $2 \%$ to $32 \%$.

Figure 2 shows the standardised mortality ratio for all causes and certain specific causes of death. The rectangles are arranged in blocks of four, the upper pair in each block relating to males and the lower pair to females. The rectangles on the left hand side relate to those under the age of 65 years and on the right to older persons. It should be noted that standardisation, in five-year age bands, has been conducted within both the $0-64$ years and 65 years and over age groups. The figure shows that all-cause standardised mortality for this area is in the highest decile-in fact it is almost the worst area in Greater Glasgow-for males and females, both in the 0 to 64 years and 65 years and over age groups. Standardised mortality is also relatively high in all four age and sex groups for deaths from neoplasms, and for other causes of death in most age-sex groups; the actual SMR values are given in parentheses, although for some of the cause-specific groups these are based on relatively small numbers.

Figure 3 shows that for hospital admissions (linked episodes, standardised for age and sex) Bridgeton ranks relatively high - and often very high indeedfor all causes analysed. The values are highest for lung
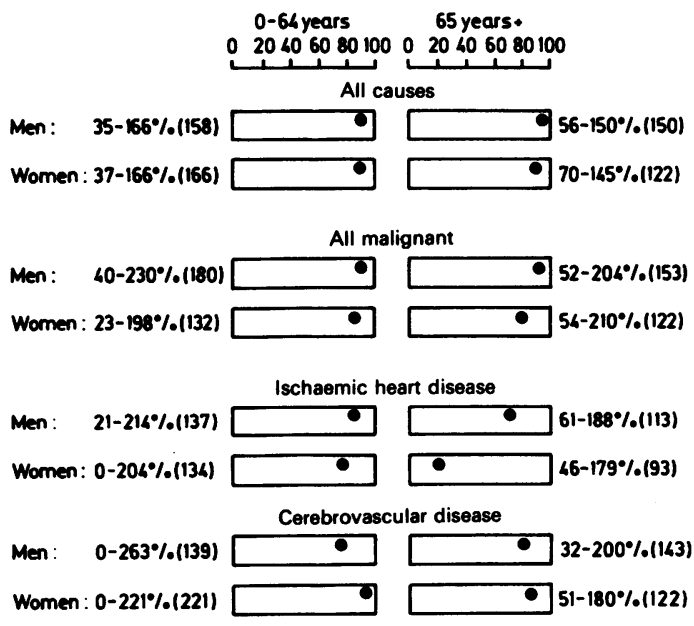

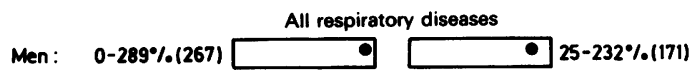

Fig 2 Standardised mortality ratios (1980-2) for males and females aged 0 to 64 years and 65 years and over: all cause mortality, and deaths from malignant neoplasms, ischaemic heart disease, cerebrovascular disease, and respiratory disease. Key as for Fig 1 .
John Womersley and Douglas McCauley

$0-64$ years 65 years.

020406080100020406080100

Lung cancer

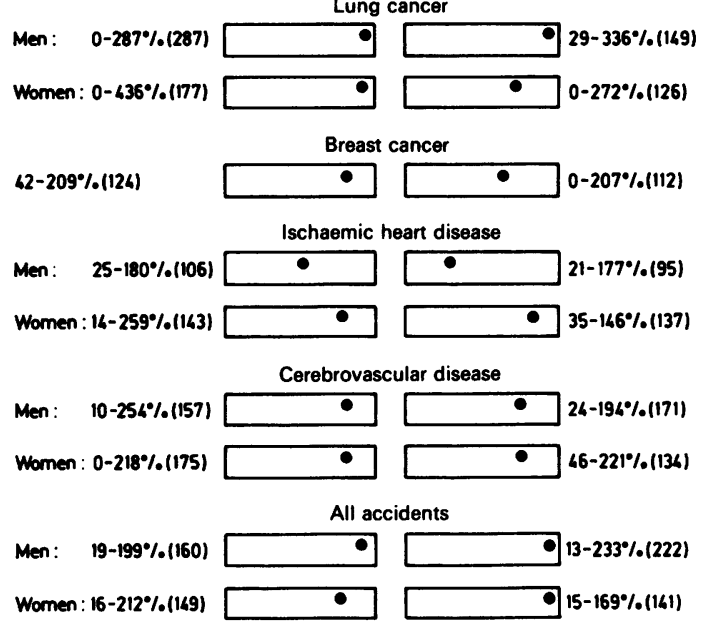

Fig 3 Standardised linked hospital discharge ratios (1980-2) for males and females aged 0 to 64 years and 65 years and over: lung cancer, breast cancer, ischaemic heart disease, cerebrovascular disease, and accidents. Key as for Fig 1.

cancer in younger men and women and for stroke and accidents in all four sex and age groups.

Figure 4 shows that Bridgeton is in the highest decile of areas in its proportions of babies which are of low birthweight, are born to single parents, and are not breast fed; it is also in the highest decile for the proportion of unimmunised children (against diphtheria, pertussis, and measles) and of births to teenage mothers.

Figure 5 shows that Bridgeton is again in the highest deciles in the proportion of infants who are admitted to hospital, for failure to attend any doctor for developmental assessment (it is the highest in Glasgow in these respects), and for failure to attend or irregular attendance at child health clinics. Referrals made by the health visitor are slightly above average, but referrals specifically for suspected developmental delay are-perhaps surprisingly-below average. However, possibly to try to help resolve some of the problems of children in this area, health visiting rates are among the highest for any postcode sector in the Health Board-both on average (eight visits during the first 9 months of life) and in the proportions of children who receive less than four or more than nine visits during this period ( $13 \%$ and $37 \%$ respectively).

Figure 6 is a histogram for all postcode sectors within the GGHB area, showing admissions to hospital during the first 9 months of life. The proportion of children admitted to hospital during the first year of life is in the range $2-14 \%$ for infants 


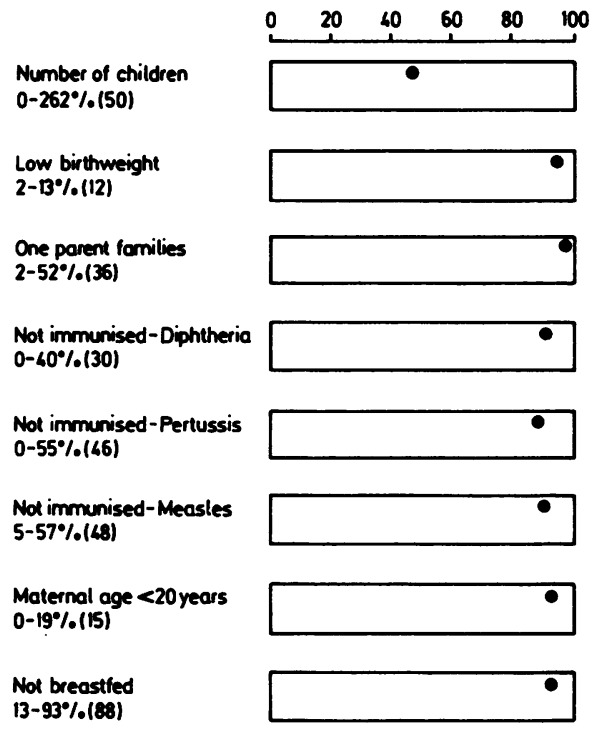

Fig 4 Child health characteristics at 9 months of age for children living in Bridgeton: number of children born in 1983; percentages birthweight $2500 \mathrm{~g}$ or below, living with a single parent, not immunised against diphtheria, pertussis, and tetanus (as of July 1985), born to teenage mothers, and not breastfed. Key as for Fig 1.

resident in most postcode sectors. The asterisk indicates the position of postcode sector G40.3although any postcode sector can be selected-and shows that between 20 and $23 \%$ of infants were admitted to hospital during their first year. It is also possible for the computer to indicate which postcode sectors lie in any part of the distribution: for example, those in which infant hospital admission rates are in the same range, $20-23 \%$, include also $G 4 \cdot 1$ and $G 32 \cdot 6$.

\section{Discussion}

The health profiles are designed to demonstrate, for each community or geographical area within a health authority, a wide range of health characteristics in a form that can be reasonably easily assimilated. Scrutiny of the health profiles for areas with particularly high standardised mortality ratios (SMRs) shows that these are also the areas with the least favourable socioeconomic characteristics, the highest hospital admission rates, and the poorest child health characteristics. Conversely, areas with low SMRs are the most affluent areas, most or all health characteristics being well above average.

The Resource Allocation (for England and Wales) and Scottish Health Authorities Revenue Equalisation Working Parties derived formulae ${ }^{1920}$ which are used to allocate revenue resources to health
Number of children $0-262 \%(50)$

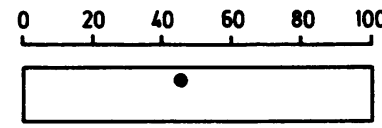

Admitted to hospital $2-23 \%(23)$

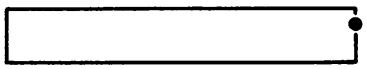

No developmental assessment $0-39 \%$ (39)

Referrals-Developmental delay $0-20 \%$ (5)

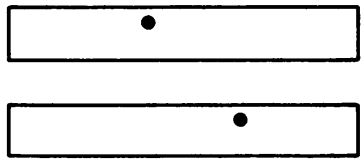

Other HV referrals

$2-86 \%$ (29)

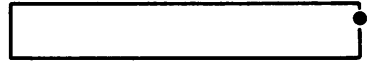

Never/Rarely go to CHC $6-62 \%(61)$

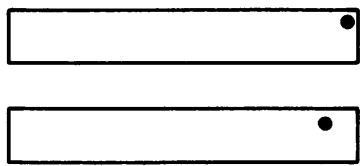

Average No. $\mathrm{HV}$ visits $5-9 \%(8)$

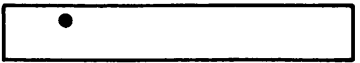

Useful HV visits $<=4$ $0-63 \%(13)$

Useful HV visits $>=9$ $2-62 \%(37)$

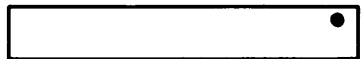

Fig 5 Child health characteristics at 9 months of age for children living in Bridgeton: number of children born in 1983; percentages admitted to hospital, not attending any doctor for developmental assessment, referred by health visitor for suspected developmental delay, referred by health visitor for other reasons, never or rarely attending a child health clinic; average number of 'successful' visits paid by the health visitor to the child, and percentages of children receiving less than five visits and more than eight visits from the health visitor. Key as for Fig 1 .

authorities according to various indicators of need. One of these indicators is the SMR for the population served by a health authority (restricted in Scotland to the 0-64 age group). Health authorities with a high population SMR therefore receive an increased allocation of resources in order to provide these extra services, and it is estimated that the Greater Glasgow Health Board-with a population SMR (0-64 years) of about $116^{*}$-derives about $7 \%$ of its total revenue allocation for community services on the basis of the SMR. It seems only fair that this extra resource should be distributed according to measures of health rather than equally among all communities within the area; if the budget is not allocated in this way, then communities with low SMRs will receive services which are made available only because other communities in the same health authority have relatively high SMRs.

* SMR for Scotland $=100$. 


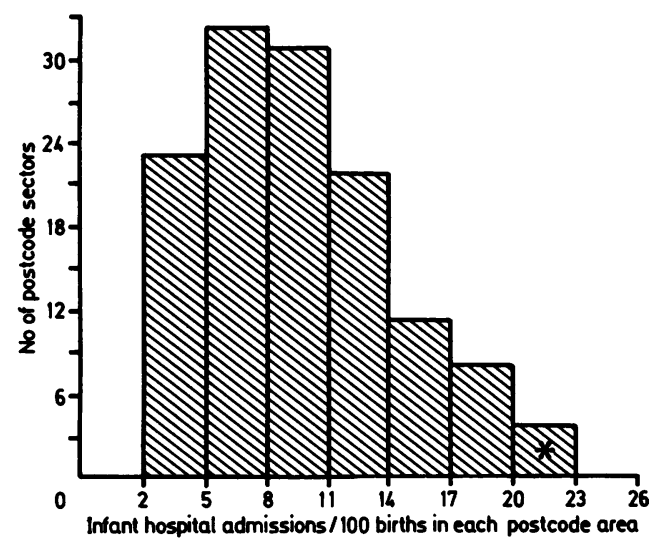

Fig 6 Histogram for all postcode sectors within Greater Glasgow showing infant hospital admissions as a percentage of total births to residents of each postcode sector. The asterisk refers to position of Bridgeton (postcode sector G40.3showing a very high rate for hospital admissions).

Further support for this suggestion is provided by Alwyn Smith, who has argued that "the unequal distribution of health can only be defended by those who believe that health ought to be unequally distributed as part of the system of incentives that drives a competitive economy". ${ }^{21}$ An important step in reducing this inequality would be to ensure that the population living in areas with high SMRs actually receive more preventive and other community services. This would be the most effective way of improving overall health, because it is easier to improve health among those whose health is poor than among those whose health is good. ${ }^{21}$

It is probably inappropriate for health services on their own to adopt a policy of discriminating in favour of areas which have been designated by other agencies as multiply deprived-firstly, because many individuals with health problems reside outwith these areas and, secondly, because the health service is unable to ameliorate the fundamental causes of deprivation. Strategies based on geographical areas and on the needs of individuals or particular groups should therefore complement one another; and integrated action in deprived areas by local government and health and other agencies is to be encouraged since improvements in employment, housing, and the environment are fundamental if health in these areas is to be improved to a significant extent.

Health services can, however, make some contribution on their own to improving health in areas where health is poorest: but merely increasing resources in, or redistributing resources in favour of, a particular area without reference to specific objectives is unlikely to be productive. The health service has effective systems for monitoring specific health needs, and health profiles such as we have described may be used to provide some indication of what services are required by which communities. For some measures, such as immunisation status, it is possible that an improvement may be achieved at relatively little cost simply by increasing publicity and providing parents and staff with more information. For other childhood measures, however, it will probably be necessary first to discover by investigation why the problem (eg, defaulting or high hospital admission rates) exists, and then to experiment with ways of alleviating it on a limited basis. In this way innovative health promotional measures would be conducted initially in one or two relatively small areas, and their success would be assessed by comparison with control areas; this should be possible over a relatively short time period, because accurate denominator data (the number of births in each postcode sector) are available each year. The information available in the health profiles should be adequate to permit such evaluation at little additional cost, and this should prevent resources being dissipated in programmes which have not been evaluated and which may be of minimal effectiveness.

For the adult measures an appropriate programme of action is less easy to define. One example of possible action has been taken by the Greater Glasgow Health Board, which has recently launched a campaign aimed at the reduction of cardiovascular morbidity and mortality by $10 \%$ over the next decade, and this will be focussed initially on areas with high standardised mortality rates. The thrust of this campaign will be directed to encouraging all adults of middle age to attend their general practice for screening for hypertension, overweight, and blood lipid abnormalities; and to encouraging a reduction in smoking and fat intake and an increase in physical activity. It is hoped that these efforts will lead to a reduction in hospital admissions and mortality from cardiovascular disease and lung cancer in those areas where these measures are least favourable, although any improvement will be more difficult to monitor than is the case for the measures of child health because of the infrequent availability of reliable population denominator data.

We conclude that the Resource Allocation Working Party (RAWP) and Scottish Health Authorities Revenue Equalisation (SHARE) formulae imply that community resources should be allocated within health authorities in favour of communities and individuals with the poorest health indicators; that community resources should be targeted according to objectively measured needs in order to achieve 
measurable improvements; that innovative programmes of health promotion are required in order to achieve many of these objectives; and that careful evaluation should be an essential component of all such programmes.

\section{References}

${ }^{1}$ Cowan R. Vital statistics of Glasgow illustrating the sanitary condition of the population. Paper read before the statistical section of the British Association, 21 September 1840.

2 Reports of the Medical Officers of Health for Glasgow, 1863-1972.

${ }^{3}$ Royal Commission on the National Health Service. Report. London: HMSO, 1979.

${ }^{4}$ Department of Health and Social Security. Inequalities in health. London: DHSS, 1980. (Black report).

5 Jarman B. A survey of primary care in London. London: Royal College of General Practitioners, 1981. (Occasional paper No 16).

${ }^{6}$ London Health Planning Consortium, Primary Health Care Study Group. Primary health care in inner London. London: DHSS, 1981. (Acheson report).

${ }^{7}$ Downham M A P S, MacGibbon R, Preston G M, Tyrell $S$ M. Medical care in the inner cities. $B r$ Med $J$ 1978; ii: 545-8.

${ }^{8}$ Wood J. Are the problems of primary care in inner cities fact or fiction? $\mathrm{Br} \mathrm{Med} J$ 1983; 286: 1109-12.
${ }^{9}$ Hume D, Womersley J. Analysis of death rates in the population aged 60 years and over of Greater Glasgow Health Board by postcode sector of residence. J Epidemiol Community Health 1985; 39: 357-63.

${ }^{10}$ Webber $R$. Census enumeration districts: a socioeconomic classification. OPCS Occasional Paper 14, 1979: London: OPCS.

${ }^{11}$ CACI (Computer and Communications Inc) Target Marketing: a classification of residential neighbourhoods (ACORN). CACI Inc., 289 High Holborn, London WCIA $7 \mathrm{HZ}$.

12 Jarman B. Identification of underprivileged areas. $\mathrm{Br} \mathrm{Med}$ $J$ 1983; 287: 130-1.

${ }^{13}$ Scott Samuel A. Need for primary health care: an objective indicator. Br Med J 1984; 288: 457-8.

14 Carstairs V. Multiple deprivation and health state. Community Medicine 1981; 3, 4-13.

15 McLure A, Stewart G T. Admission of children to hospitals in Glasgow: relation to unemployment and other deprivation variables. Lancet 1984; ii: 682-685.

16 Welsh Health Technical Services Organisation. Child Health Computing Committee record system. Whitchurch, Cardiff: Technical Services Organisation, 1983.

${ }^{17}$ McIntosh H T, Womersley J. The Glasgow health visitor developmental screening module. Community Med 1986; 8: 111-23.

18 Department of Health and Social Security. Performance indicators. London: DHSS, Performance Indicator Branch, Euston Tower, 1985.

19 Department of Health and Social Security. First interim report of the Resource Allocation Working Party. Allocations to regions in 1976/77. London: DHSS, 1975.

${ }^{20}$ Scottish Home and Health Department. Scottish Health Authorities Revenue Equalisation (SHARE). Report of the Working Party on Resource Allocation. Edinburgh: HMSO, 1977.

${ }^{21}$ Smith A. The functions of a health service. Lancet 1985; (i): 806-808. 